\title{
Propagation Mechanism Analysis before the Break Point inside Tunnels
}

\author{
Ke Guan*, Zhangdui Zhong*, Bo Ai* and Cesar Briso-Rodríguez ${ }^{\dagger}$ \\ ${ }^{*}$ State Key Laboratory of Rail Traffic Control and Safety \\ Beijing Jiaotong University, 100044, Beijing, China \\ †Escuela Universitaria de Ingeniería Técnica de Telecomunicación \\ Universidad Politécnica de Madrid, 28031, Madrid, Spain \\ E-mail: myecone@hotmail.com
}

\begin{abstract}
There is no unanimous consensus yet on the propagation mechanism before the break point inside tunnels. Some deem that the propagation mechanism follows the free space model, others argue that it should be described by the multimode waveguide model. Firstly, this paper analyzes the propagation loss in two mechanisms. Then, by conjunctively using the propagation theory and the three-dimensional solid geometry, a generic analytical model for the boundary between the free space mechanism and the multi-mode waveguide mechanism inside tunnels has been presented. Three measurement campaigns validate the model in different tunnels at different frequencies. Furthermore, the condition of the validity of the free space model used in tunnel environment has been discussed in some specific situations. Finally, through mathematical derivation, the seemingly conflicting viewpoints on the free space mechanism and the multi-mode waveguide mechanism have been unified in some specific situations by the presented generic model. The results in this paper can be helpful to gain deeper insight and better understanding of the propagation mechanism inside tunnels.

Index Terms-boundary; break point; mechanism; modeling; propagation; tunnel
\end{abstract}

\section{INTRODUCTION}

For a reliable wireless communication system (e.g. CBTC [1], DSRC [2]) design a precise propagation model is mandatory, so that the required system parameters can be predicted in advance without the need of expensive, site specific measurements. It is noteworthy that without a correct comprehension of the propagation mechanism it is impossible to build a reliable model. Thus, holding the propagation mechanism is essential to establish accurate model. Since the tunnel, both the railway tunnel and the road tunnel, is a common and complex propagation scenario, a great number of propagation models inside tunnels have been presented since seventies of last century. These models revealed that there exists a "critical distance" [3][4], usually called as the break point [3][4][5]. Before the break point is the near-field region, where the high order modes are significant; guided propagation has not been

This work is supported by the NNSF of China under Grant 60830001, Program for New Century Excellent Talents in University under Grant NCET09-0206, Beijing NSF 4112048, the Key Project of State Key Lab. of Rail Traffic Control and Safety under Grant RCS2008ZZ006, Program for Changjiang Scholars and Innovative Research Team in University under Grant No. IRT0949 and the State Key Lab. of Rail Traffic Control and Safety (Contract No. RCS2008ZT005, No. RCS2010ZT012 and No. RCS2010K008), Beijing Jiaotong University. well established and suffers larger loss. After the break point is the far-field region, in which the high order modes have been greatly attenuated; guided propagation has been stabilized and undergoes smaller loss [3][4][6][8].

The propagation characteristics in the far-field region inside tunnels follow the fundamental-mode waveguide mechanism [3][4][8][9]; nevertheless, there is no unanimous consensus yet on the propagation mechanism before the break point within tunnels. Some dissertations are inclined to interpret the propagation in the near-field region with the single ray (free space) theory [8][9][10], others contend that it should be described by the multi-mode waveguide model [3][4][11]. A great deal of evidence proves that both these two different propagation mechanisms exist before the break point inside tunnels. Hence, to reveal the propagation mechanism situation in the near-field region it is essential to model the accurate location of the boundary between the free space mechanism and the multimode waveguide mechanism. This is important since along with the increase of operating frequency in advanced radio communication system utilized inside tunnels, the location of break point is more and more far away from the transmitter and this means the main effective propagation area inside tunnels is before the break point.

\section{AnAlytical Modeling for the Propagation MECHANISMS AND THEIR BOUNDARY}

In this paper, the propagation loss is modeled by modal theory; the location of the boundary between two propagation mechanisms is modeled by the combination of geometry and propagation theory.

\section{A. Geometrical and Electrical Modeling for the tunnel}

Although the arched tunnel is the most common type, the field variation inside arched tunnels can be predicted with sufficient accuracy by assuming a rectangular tunnel [12][13]. Hence, in this paper, the tunnel cross section is treated as an equivalent rectangle. To use the geometrical and modal analysis, following parameters are required:

- Coordinate system: Three-dimensional Cartesian coordinate system, with its origin located at an angle of the rectangle tunnel. 
- Geometric dimension: width of rectangular tunnel:a; height of rectangular tunnel: $b$; length of rectangular tunnel: $c$. The coordinates of transmitter, receiver and the middle point on the line of sight between transmitter and receiver are $P_{t}\left(x_{t}, y_{t}, z_{t}\right), P_{r}\left(x_{r}, y_{r}, z_{r}\right)$ and $P_{0}\left(x_{0}, y_{0}, z_{0}\right)$; their relationships are expressed by

$$
x_{0}=\frac{x_{r}+x_{t}}{2}, y_{0}=\frac{y_{r}+y_{t}}{2}, z_{0}=\frac{z_{r}+z_{t}}{2}
$$

- Electrical parameters: relative permittivity for vertical and horizontal walls: $\varepsilon_{v}$ and $\varepsilon_{h}$, with the typical values for concrete: $\varepsilon_{v}=\varepsilon_{h}=5$ [3].

\section{B. Propagation Loss in Different Propagation Mechanisms}

- Propagation loss in the free space propagation segment. In the adjacent region of the transmitter antenna, the angles of incidence from the ray to the wall (vertical and horizontal) are high resulting in high attenuation of reflected rays, whereas the path difference between direct and reflected rays may also cause additional attenuation, thus only the direct ray significantly contributes to the strength of the received signal. The channel loss in this segment follows the free space loss attenuation [14]

$$
P L(d B)=-10 \log _{10}\left[\frac{\lambda^{2}}{(4 \pi)^{2}\left|z_{r}-z_{t}\right|^{2}}\right]
$$

where $\left|z_{r}-z_{t}\right|$ is the distance between the transmitter and receiver in metres, and $\lambda$ is the signal wavelength.

- Propagation loss in the multi-mode waveguide segment. According to the modal theory, rectangular tunnel can be regarded as an oversized imperfect hollow rectangular waveguide. Since the UHF is considerably higher than the cutoff frequency of the fundamental modes which is very low, a wide range of $E_{m n}$ multiple modes propagate inside tunnels when the free space segment ends [9]. Using modal theory, the losses of horizontally and vertically polarized $E_{m n}$ modes can be given by

$$
\begin{aligned}
& \alpha(m, n)^{h}=4.343 \lambda^{2}\left(\frac{m^{2} \varepsilon_{v}}{a^{3} \sqrt{\varepsilon_{v}-1}}+\frac{n^{2}}{b^{3} \sqrt{\varepsilon_{h}-1}}\right) d B / m \\
& \alpha(m, n)^{v}=4.343 \lambda^{2}\left(\frac{m^{2}}{a^{3} \sqrt{\varepsilon_{v}-1}}+\frac{n^{2} \varepsilon_{h}}{b^{3} \sqrt{\varepsilon_{h}-1}}\right) d B / m
\end{aligned}
$$

With the propagation constants offered above, the propagation loss in the multi-mode waveguide segment can be calculated considering the modes for both polarizations

$$
\begin{aligned}
& L_{m n}^{v / h}(d B)= \\
& 10 \lg \left[\sum_{i=1}^{m} \sum_{j=1}^{n} \times \sqrt{10^{2 \alpha(i, j)^{h}\left|z_{r}-z_{t}\right|}+10^{2 \alpha(i, j)^{v}\left|z_{r}-z_{t}\right|}}\right]
\end{aligned}
$$

\section{Boundary between Different Propagation Mechanisms}

To hold the accurate propagation mechanism situation, it is essential to model the location of the boundary between the free space propagation segment and the multi-mode waveguide segment. Fig. 1 depicts the three-dimensional geometry schematic diagram of the tunnel, transmitter, receiver, line of sight and the Maximum first Fresnel zone.

By utilizing the Lagrange multiplier method seeking extreme conditions, the distance between a point $P\left(x_{0}, y_{0}, z_{0}\right)$ and a line $l:\left\{\begin{array}{l}A_{1} x+B_{1} y+C_{1} z=0 \\ A_{2} x+B_{2} y+C_{2} z=0\end{array}\right.$ in three-dimensional space can be given by

$$
\begin{aligned}
& d= \\
& \frac{\left|\left(A_{1} x_{0}+B_{1} y_{0}+C_{1} z_{0}+D_{1}\right) \vec{n}_{2}-\left(A_{2} x_{0}+B_{2} y_{0}+C_{2} z_{0}+D_{2}\right) \vec{n}_{1}\right|}{\left|\vec{n}_{1} \times \vec{n}_{2}\right|}
\end{aligned}
$$

where $\vec{n}_{i}=\left|A_{i}, B_{i}, C_{i}\right|,(i=1,2)$.

Thus, let the wall of the tunnel expressed by a plane in general type as

$$
A x+B y+C z+D=0
$$

the tangent line between the Maximum Fresnel zone plane and the wall can be written as

$$
\left\{\begin{array}{c}
\left(x_{r}-x_{t}\right)\left(x-\frac{x_{r}+x_{t}}{2}\right)+\left(y_{r}-y_{t}\right)\left(y-\frac{y_{r}+y_{t}}{2}\right) \\
+\left(z_{r}-z_{t}\right)\left(z-\frac{z_{r}+z_{t}}{2}\right)^{2}=0 \\
A x+B y+C z+D=0
\end{array}\right.
$$

Then,

$$
\begin{gathered}
\vec{n}_{1}=\left\{\left(x_{r}-x_{t}\right),\left(y_{r}-y_{t}\right),\left(z_{r}-z_{t}\right)\right\} \\
\vec{n}_{2}=\{A, B, C\}
\end{gathered}
$$

Let $\delta_{1}=A_{1} x_{0}+B_{1} y_{0}+C_{1} z_{0}+D_{1}$, combining (1), $\delta_{1}$ can be rewritten as

$$
\begin{aligned}
\delta_{1}= & \left(x_{r}-x_{t}\right) \cdot \frac{x_{r}+x_{t}}{2}+\left(y_{r}-y_{t}\right) \cdot \frac{y_{r}+y_{t}}{2} \\
& +\left(z_{r}-z_{t}\right) \cdot \frac{z_{r}+z_{t}}{2}-\left(\frac{x_{r}^{2}-x_{t}^{2}}{2}+\frac{y_{r}^{2}-y_{t}^{2}}{2}+\frac{z_{r}^{2}-z_{t}^{2}}{2}\right)
\end{aligned}
$$

Similarly,

$$
\begin{aligned}
\delta_{2} & =A_{2} x_{0}+B_{2} y_{0}+C_{2} z_{0}+D_{2} \\
& =A \cdot \frac{x_{r}+x_{t}}{2}+B \cdot \frac{y_{r}+y_{t}}{2}+C \cdot \frac{z_{r}+z_{t}}{2}+D
\end{aligned}
$$

Thus, according to (6), combining (7)-(12), the distance between the middle point $P_{0}\left(x_{0}, y_{0}, z_{0}\right)$ on the line of sight and the tangent line between the Maximum Fresnel zone plane and the wall of tunnel can be obtained as

$$
d=\frac{\left|\delta_{1} \cdot \vec{n}_{2}-\delta_{2} \cdot \vec{n}_{1}\right|}{\left|\vec{n}_{1} \times \vec{n}_{2}\right|}
$$

where

$$
\begin{aligned}
& \left|\delta_{1} \cdot \vec{n}_{2}-\delta_{2} \cdot \vec{n}_{1}\right|= \\
& \quad \sqrt{\left[\delta_{2}\left(x_{r}-x_{t}\right)\right]^{2}+\left[\delta_{2}\left(y_{r}-y_{t}\right)\right]^{2}+\left[\delta_{2}\left(z_{r}-z_{t}\right)\right]^{2}} \\
& \left|\vec{n}_{1} \times \vec{n}_{2}\right|=\left\{\left[\left(y_{r}-y_{t}\right) C-\left(z_{r}-z_{t}\right) B\right]^{2}+\right. \\
& \left.\left[\left(x_{r}-x_{t}\right) C-\left(z_{r}-z_{t}\right) A\right]^{2}+\left[\left(x_{r}-x_{t}\right) B-\left(y_{r}-y_{t}\right) A\right]^{2}\right\}^{\frac{1}{2}}
\end{aligned}
$$

Based on the propagation theory, the radius of the first Fresnel zone is determined by

$$
r_{1}=\sqrt{\frac{\lambda d_{1} d_{2}}{d_{1}+d_{2}}}
$$

where $d_{1}$ denotes the distance between the transmitter and the interaction between the line of sight and the first Fresnel 
zone, $d_{2}$ denotes the distance between the receiver and the interaction. When the interaction is the middle point $P_{0}, d_{1}=$ $d_{P_{t} P_{0}}=d_{2}=d_{P_{0} P_{r}}=\frac{1}{2} d_{P_{t} P_{r}}$. At this point, the radius gets the maximum value of the first Fresnel zone

$$
\begin{aligned}
& r_{1 \text { Max }}=\frac{1}{2} \sqrt{\lambda d_{P_{t} P_{r}}} \\
& =\frac{1}{2} \sqrt{\lambda \sqrt{\left(x_{r}-x_{t}\right)^{2}+\left(y_{r}-y_{t}\right)^{2}+\left(z_{r}-z_{t}\right)^{2}}}
\end{aligned}
$$

Propagation theory indicates that the free space loss channel model can be applied if the first Fresnel zone is free of any obstacles. Therefore, if only one wall of the tunnel could be touched by the Maximum first Fresnel zone, the boundary between two propagation mechanisms locates at $z_{r}\left(d_{i}\right)$ when

$$
d_{i}=r_{1 M a x}, i=C, F, L, R
$$

is satisfied, in which $d_{C}, d_{F}, d_{L}$ and $d_{R}$ denotes the distance between the circle center of the Maximum first Fresnel zone $P_{0}$ and the tangent point $P_{C}, P_{F}, P_{L}$ and $P_{R}$, respectively. However, in fact, there are totally four walls of the tunnel (the ceiling, the floor, the right wall and the left wall) which could be tangent to the the Maximum first Fresnel zone. Hence, the boundary between two propagation mechanisms locates at $z_{r}$ when the Maximum first Fresnel zone first touches any one of the walls. Thus, the boundary locates at

$$
z_{r}=\operatorname{Min}\left\{z_{r}\left(d_{i}\right) \mid d_{i}=r_{1 M a x}, z_{r} \in R^{+}\right\}, i=C, F, L, R
$$

\section{BOUNDARY MODEL VALIDATION}

Three groups of measurement campaigns have been employed for validating the model of the boundary presented in the previous section. The first set of measurements was carried out in a railway tunnel in Spain at $900 \mathrm{MHz}$ [3]. According to the configuration of the test, following parameters in the boundary model can be estimated as: $a=10.7, b=6.3$, $x_{t}=0.2, y_{t}=4, z_{t}=0, x_{r}=3, y_{r}=3$. By solving (18) and using (19), the accurate distance of the boundary is obtained: $z_{r}=30.86$. As shown in Fig.2, the boundary separates two propagation segments clearly. The propagation in the segment before the boundary follows the free space mechanism and the free space loss model has a good agreement with the measured data. The segment after the boundary is dominated

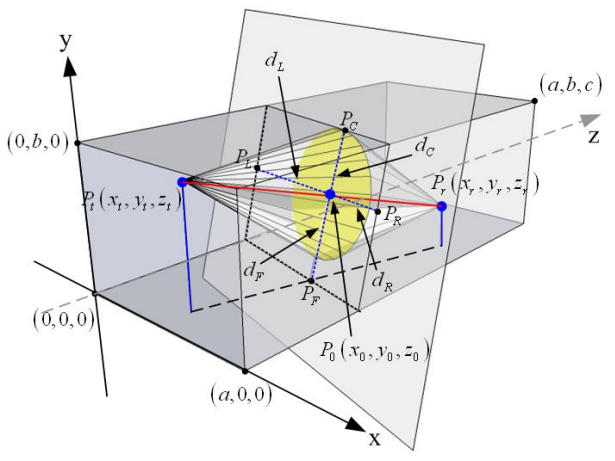

Fig. 1. Schematic diagram of the propagation inside tunnels with the first Fresnel zone clearance.

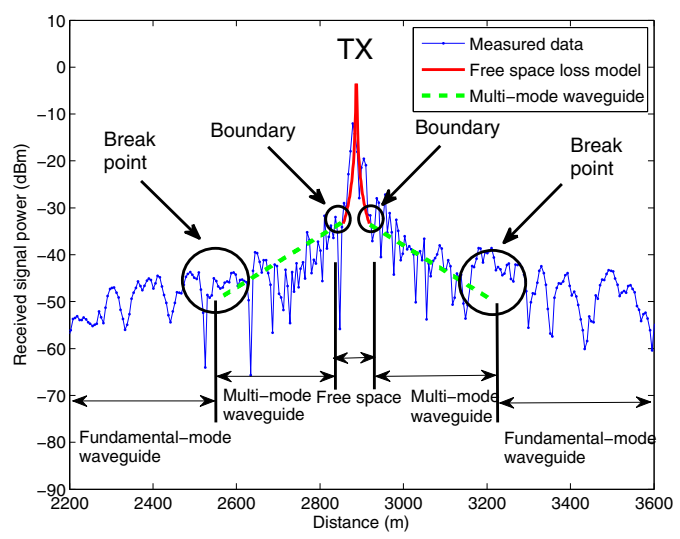

Fig. 2. Propagation mechanisms and their boundaries before the break point inside tunnels.

TABLE I

COMPARISONS BETWEEN THE MODEL AND THE MEASUREMENTS ON THE BOUNDARY BETWEEN TWO PROPAGATION MECHANISMS

\begin{tabular}{cccc}
\hline Tunnel & Frequency & Measured & modeled \\
\hline Railway tunnel [3] & $0.9 \mathrm{GHz}$ & $30-35 \mathrm{~m}$ & $30.86 \mathrm{~m}$ \\
Railway tunnel [9] & $0.4 \mathrm{GHz}$ & $15 \mathrm{~m}$ & $13.65 \mathrm{~m}$ \\
Road tunnel [9] & $0.4 \mathrm{GHz}$ & $15 \mathrm{~m}$ & $15.41 \mathrm{~m}$ \\
\hline
\end{tabular}

by the multi-mode waveguide mechanism and the multi-mode waveguide loss model also shows a reasonable performance.

The second group of measurements was performed in a railway tunnel typical to Europe at $400 \mathrm{MHz}$ [9]. Here are the parameters in the measurement: $a=4.7, b=4.5$, $x_{t}=2.35, y_{t}=1.5, z_{t}=0, x_{r}=2.35, y_{r}=1.7$. By solving (18) and using (19), the final distance of the boundary is obtained as: $z_{r}=13.65$. The third set of measurements was taken in a dual-carriageway road tunnel linking Austria and Slovenia also at $400 \mathrm{MHz}$ [9]. Following the parameters in the measurement: $a=9.5, b=4.7, x_{t}=2.5, y_{t}=1.7$, $z_{t}=0, x_{r}=2.5, y_{r}=1.7$. The distance of the boundary derived by the model is : $z_{r}=15.41$. Table I shows the global comparisons between the results of the model and the measurements on the boundary. The validation results indicate that the boundary model presented in this paper is valid and easy to be used.

\section{Boundary Model in Certain Specific Situations}

Several publications, e.g. [9], [10], etc., employed the model in the free space to calculate the boundary as

$$
z_{r}=\frac{4 y_{r} y_{t}}{\lambda}
$$

Some literatures, such as [8], yielded the end of the free space segment as

$$
z_{r}=\operatorname{Max}\left(\frac{a^{2}}{\lambda}, \frac{b^{2}}{\lambda}\right)
$$

whereas some dissertations ([3], [4], [11], etc.) used the same equation upon to indicate the end of the multi-mode waveguide segment. 


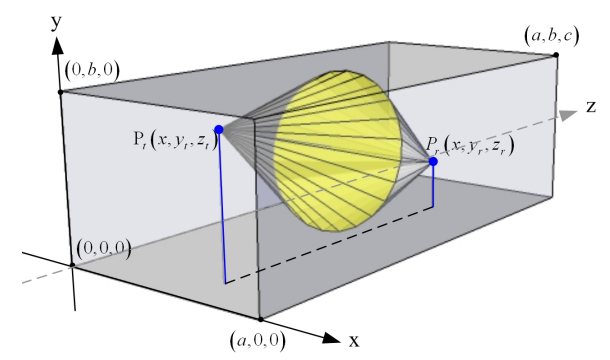

Fig. 3. Special Situation One: $x_{t}=x_{r}=x$.

In fact, these seemingly different and conflicting views are all reasonable, but not universal, as their validity requires certain conditions. What follows are some discussions about these conditions based on the simplification of the boundary model in specific situations.

\section{A. Specific Situation One}

In some cases, such as CBTC [1], the communication is between the transmitter installed under the ceiling and the receiver on-train in the railway tunnel or the subway tunnel, i.e. $x_{t}=x_{r}=x$, as shown in Fig. 3. In this situation, the analytic solution of (18) can be given by

$$
\begin{gathered}
z_{r}\left(d_{L}\right)=\frac{\sqrt{16 x^{4}-\lambda^{2}\left(y_{r}-y_{t}\right)^{2}}}{\lambda} \\
z_{r}\left(d_{R}\right)=\frac{\sqrt{16(x-a)^{4}-\lambda^{2}\left(y_{r}-y_{t}\right)^{2}}}{\lambda}
\end{gathered}
$$

If $y_{r} \approx y_{t}$, then

$$
\begin{gathered}
z_{r}\left(d_{F}\right) \approx \frac{4 y_{r} y_{t}}{\lambda} \\
z_{r}\left(d_{C}\right) \approx \frac{4\left(b-y_{r}\right)\left(b-y_{t}\right)}{\lambda}
\end{gathered}
$$

It is noteworthy that (24) is the model in the free space, and (25) can be seen as a transformation of the free space model as the difference is only the floor replaced by the ceiling.

Thus, in the Specific Situation One, the conditions of the validity of the free space model or the transformation model used in tunnel environment are:

- $y_{r} \approx y_{t}$ : the heights of transmitter and receiver are approximate.

- $\operatorname{Min}\left\{y_{t}, y_{r}, b-y_{t}, b-y_{r}\right\} \leq \operatorname{Min}\{x, a-x\}$ : the distance between ceiling/floor and transmitter/receiver is shorter than between right/left wall and transmitter/receiver.

More specifically, the criterion of determining the employment of the free space model or its transformation are:

- When $\operatorname{Min}\left\{y_{t}, y_{r}\right\} \geq \operatorname{Min}\left\{b-y_{t}, b-y_{r}\right\}, z_{r} \approx$ $\frac{4\left(b-y_{r}\right)\left(b-y_{t}\right)}{\lambda}$, since $d_{C}=\left(b-\frac{y_{r}+y_{t}}{2}\right) \sec \theta \leq$ $\left(\frac{y_{r}+y_{t}}{2}\right) \sec \theta=d_{F}$, as shown in Fig. 4.

- When $\operatorname{Min}\left\{y_{t}, y_{r}\right\} \leq \operatorname{Min}\left\{b-y_{t}, b-y_{r}\right\}, z_{r} \approx$ $\frac{4 y_{r} y_{t}}{\lambda}$, since $d_{C}=\left(b-\frac{y_{r}+y_{t}}{2}\right) \sec \theta \geq\left(\frac{y_{r}+y_{t}}{2}\right) \sec \theta=$ $d_{F}$, as shown in Fig. 5.

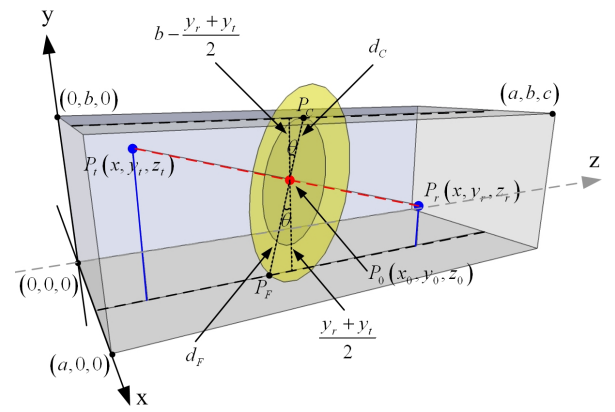

Fig. 4. Special Situation One: $\operatorname{Min}\left\{y_{t}, y_{r}\right\} \geq \operatorname{Min}\left\{b-y_{t}, b-y_{r}\right\}$.

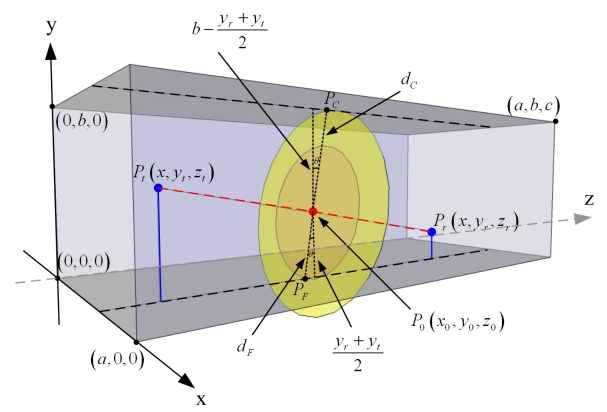

Fig. 5. Special Situation One: $\operatorname{Min}\left\{y_{t}, y_{r}\right\} \leq \operatorname{Min}\left\{b-y_{t}, b-y_{r}\right\}$.

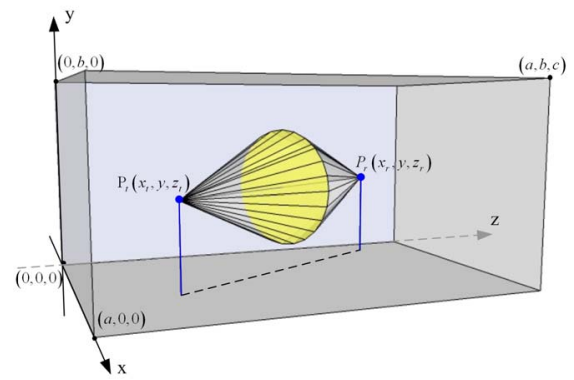

Fig. 6. Special Situation Two: $y_{t}=y_{r}=y$.

\section{B. Specific Situation Two}

In some systems, such as DSRC (Dedicated Short Range Communication) [2], the communication is among different vehicles (car or carriage). In this case, the transmitter and the receiver always have similar heights: $y_{t}=y_{r}=y$, as shown in Fig. 6. The analysis in this situation is very similar to the Specific Situation One. If $x_{r} \approx x_{t}$, then the analytic solution of (18) can be given by

$$
\begin{gathered}
z_{r}\left(d_{L}\right) \approx \frac{4 x_{r} x_{t}}{\lambda} \\
z_{r}\left(d_{R}\right) \approx \frac{4\left(a-x_{r}\right)\left(a-x_{t}\right)}{\lambda} \\
z_{r}\left(d_{F}\right) \approx \frac{4 y^{2}}{\lambda} \\
z_{r}\left(d_{C}\right) \approx \frac{4(b-y)^{2}}{\lambda}
\end{gathered}
$$

Obviously, (28) follows the model in the free space, and (29) is also a transformation of the free space model.

Thus, in the Specific Situation Two, the conditions of the validity of the free space model or the transformation model used in tunnel environment are: 


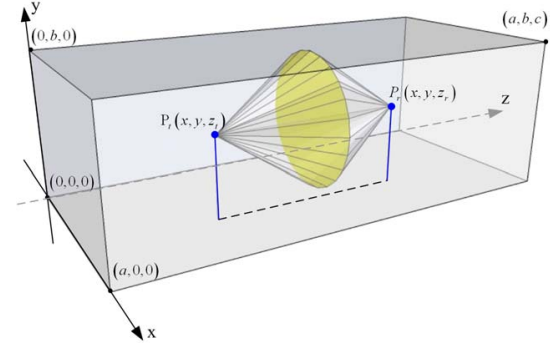

Fig. 7. Special Situation Three: $x_{t}=x_{r}=x, y_{t}=y_{r}=y$.

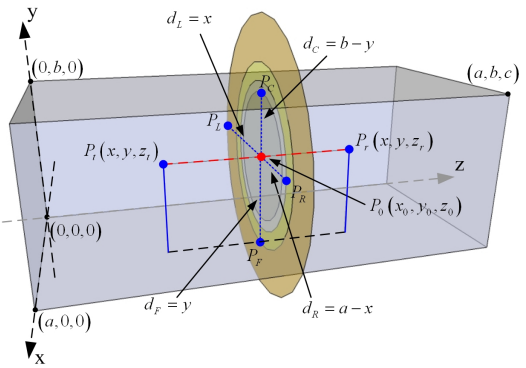

Fig. 8. Schematic diagram of the propagation in Special Situation Three.

- $x_{r} \approx x_{t}$ : the transmitter and the receiver are approximately in a line along the tunnel.

- $\operatorname{Min}\{y, b-y\} \leq \operatorname{Min}\left\{x_{t}, x_{r}, a-x_{t}, a-x_{r}\right\}$ : the distance between ceiling/floor and transmitter/receiver is shorter than between right/left wall and transmitter/receiver.

More specifically, the criterion of determining the employment of the free space model or its transformation are:

- When $y \geq b-y, z_{r} \approx \frac{4(b-y)^{2}}{\lambda}$.

- When $y \leq b-y, z_{r} \approx \frac{4 y^{2^{\lambda}}}{\lambda}$.

\section{Specific Situation Three}

In some special designed systems, the conditions in the two specific situations can be fulfilled simultaneously, that is: $x_{t}=$ $x_{r}=x, y_{t}=y_{r}=y$, as shown in Fig. 7. Through the same analysis, the analytic solution of equation (18) can be given by $z_{r}\left(d_{L}\right)=\frac{4 x^{2}}{\lambda}, z_{r}\left(d_{R}\right)=\frac{4(a-x)^{2}}{\lambda}, z_{r}\left(d_{F}\right)=\frac{4 y^{2}}{\lambda}, z_{r}\left(d_{C}\right)=$ $\frac{4(b-y)^{2}}{\lambda}$.

As illustrated in Fig. 8, in this specific situation, the condition of the validity of the free space model or the transformation model used in tunnel environment is:

$$
\text { - } \operatorname{Min}\{y, b-y\} \leq \operatorname{Min}\{x, a-x\} \text {. }
$$

More specifically, the criterion of determining the employment of free space model or its transformation are:

- When $y \geq b-y, z_{r}=\frac{4(b-y)^{2}}{\lambda}$.

- When $y \leq b-y, z_{r}=\frac{4 y^{2^{\lambda}}}{\lambda}$.

Now, if this situation is more specific:

- $x_{t}=x_{r}=\frac{a}{2}$

- $y_{t}=y_{r}=\frac{b}{2}$

the solutions can be: $z_{r}\left(d_{L}\right)=z_{r}\left(d_{R}\right)=\frac{a^{2}}{\lambda}, z_{r}\left(d_{C}\right)=$ $z_{r}\left(d_{F}\right)=\frac{b^{2}}{\lambda}$. At this moment, the distance of the end of the free space segment is

$$
z_{r}=\operatorname{Min}\left(\frac{a^{2}}{\lambda}, \frac{b^{2}}{\lambda}\right)
$$

which is different to the end of the multi-mode waveguide segment reported in [3][4][11]. Thus, even in this situation, the views from [8][9][10] and [3][4][11] still seem to be conflicting. However, if we add a condition to this situation:

$$
\text { - } a=b
$$

the conflicting views will be unified as

$$
z_{r}=\operatorname{Min}\left(\frac{a^{2}}{\lambda}, \frac{b^{2}}{\lambda}\right)=\operatorname{Max}\left(\frac{a^{2}}{\lambda}, \frac{b^{2}}{\lambda}\right)=\frac{a^{2}}{\lambda}=\frac{b^{2}}{\lambda}
$$

Thus, previous views on the propagation mechanisms and their boundaries inside tunnels are conditional valid, and even can be unified in certain specific situation; whereas the boundary model presented in this paper is general.

\section{CONCLusion}

This paper analyzes the propagation mechanism before the break point inside tunnels. A generic analytical model for the boundary between the free space propagation mechanism and the multi-mode waveguide propagation mechanism has been presented. Through the discussion on various specific situations, previous different and seemingly conflicting views have been unified by this generic model. Three sets of measurements prove that the presented model is valid and can be helpful to the accurate understanding of the propagation mechanism inside tunnels.

\section{REFERENCES}

[1] "IEEE standard for Communications-Based Train Control (CBTC) performance and functional requirements", 30. Dec. 1999.

[2] Notice of Proposed Rulemaking and Order FCC 03-324, Federal Communications Commission, Feb. 2003.

[3] C. Briso-Rodriguez, J.M. Cruz and J.I. Alonso,"Measurements and Modeling of Distributed Antenna Systems in Railway Tunnels," IEEE Trans. on Veh. Technol., vol. 56, pp. 2870-2879, Sept. 2007.

[4] Y.P. Zhang and Y. Hwang, "Enhancement of rectangular tunnel waveguide model," Microwave Conf. Proceedings 1997. APMC '97, 1997 AsiaPacific, Vol. 1, pp. 197 - 200, Dec. 1997.

[5] Y. P. Zhang and Y. Hwang, "Characterization of uhf radio propagation channels in tunnel environments for microcellular and personal communications." IEEE Trans. on Veh. Technol., Vol. 47, pp.283-296, 1998.

[6] J. Alonso, B. Izquierdo, J. Romeu, "Break point analysis and modelling in subway tunnels," 3rd European Conference on Ant. and Propag., Vol. 49, pp. 3524-3258, June. 2009.

[7] Ke Guan, Zhangdui Zhong, Bo Ai, Cesar Briso-Rodriguez, "Research of propagation characteristics of break point: near zone and far zone under operational subway condition," IWCMC, pp. 114-118, May. 2010.

[8] Y.P. Zhang, "Novel model for propagation loss prediction in tunnels,"IEEE Trans. on Veh. Technol., Vol. 52, pp. 1308 - 1314, Sept. 2003.

[9] A. Hrovat, G. Kandus, T. Javornik, "Four-slope channel model for path loss prediction in tunnels at $400 \mathrm{MHz}$, 'IET Microwaves, Antennas and Propagation, Vol. 4, pp. 571-582, Dec. 2010.

[10] Y.P. Zhang, G.X. Zheng, J.H. Sheng, "Radio propagation at $900 \mathrm{MHz}$ in underground coal mines," IEEE Trans. Antennas Propag., Vol. 49, pp. 757-762, Nov. 2001.

[11] T. Klemenschits and E. Bonek, "Radio coverage of road tunnels at 900 and $1800 \mathrm{MHz}$ by discrete antennas," 5th IEEE International Symposium on PIMRC, Vol. 2, pp. 411-415, Sep. 1994.

[12] M. Lienard and P. Degauque, "Natural wave propagation in mine environments," IEEE Trans. Antenna Propag., vol. 48, no. 9, pp. 13261339, Sep. 2000.

[13] M. Lienard and P. Degauque, "On the possibility of interpreting field variations and polarization in arched tunnels using a model for propagation in rectangular or circular Tunnels," IEEE Trans. Ant. and Propag., vol. 56, pp. 1206-1211, April. 2008.

[14] S. SAUNDERS, "Antennas and propagation for wireless communication systems," John Wiley and Sons Ltd, Chichester, England, 2005. 